
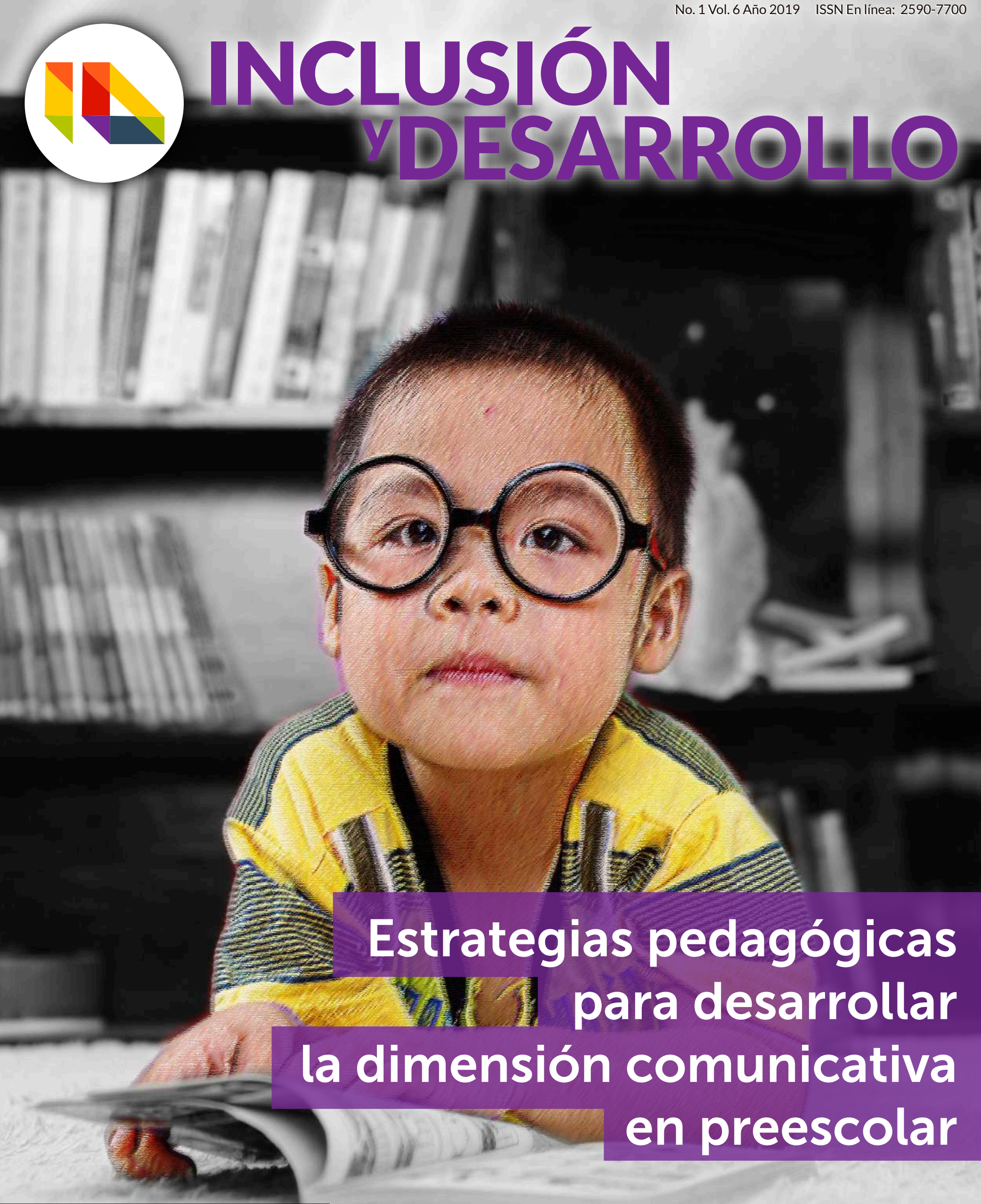

en preescolar

Yenny Andrea Perez Barrera 


\title{
ESTRATEGIAS PEDAGÓGICAS PARA DESARROLLAR LA DIMENSIÓN COMUNICATIVA EN PREESCOLAR
}

\author{
Pedagogical strategies to deVelop
} THE COMMUNICATIVE DIMENSION IN PRESCHOOL

\author{
Yenny Andrea Perez Barrera \\ perez.y@javeriana.edu.co \\ Secretaria de Educación de la Gobernación de Cundinamarca \\ Bogotá D.C. - Colombia
}

Encuentre este artículo en

http://revistas.uniminuto.edu/

Fecha de recepción: 9 de agosto de 2018

Fecha de aceptación: 8 de octubre de 2018 Fecha de publicación: 30 de diciembre de 2018

\section{Resumen}

Objetivo. Analizar cómo el docente de transición de un jardín público de Bogotá, hace uso de estrategias pedagógicas en sus clases para estimular el desarrollo de la dimensión comunicativa, a través de un aprendizaje significativo, que sea transferible a su realidad.

Metodología. Esta propuesta se estructuró partiendo de un proceso investigativo de tipo cualitativo, con la aplicación de instrumentos de recolección de datos, como: entrevista semiestructurada, observación y análisis de contenido, en un grupo de treinta y dos infantes, con edades entre los cuatro a cinco años de edad y la docente a cargo, para obtener elementos que fueron interpretados mediante el uso de una estrategia de análisis de datos, para a continuación realizar el análisis de la triangulación de datos.

Resultados. El impacto de este proyecto evidenció en primer lugar que, diseñando y generando espacios de aprendizaje significativos, los niños y niñas desarrollan capacidades comunicativas que transfieren a la realidad, permitiéndoles desenvolverse con mayor propiedad socialmente. Como segunda medida, con la implementación de estrategias pedagógicas preinstruccionales, coinstruccionales y postinstruccionales de una secuencia de enseñanza- aprendizaje, se estimula el desarrollo de habilidades y destrezas en cada una de las dimensiones del ser humano, logrando con ello un desarrollo integral, que sea útil y transferible a realidad del estudiante.

Conclusión. La implementación de estrategias pedagógicas estimula el desarrollo de habilidades y destrezas comunicativas, al generar escenarios de participación activa, en donde la construcción colectiva del saber, permite la generación de un aprendizaje significativo que sea transferible a la realidad. La aplicación de estrategias pedagógicas además de la transmisión de un conocimiento práctico, permitió el logro de un aprendizaje significativo que sobrepasó las expectativas iniciales, e invitó a seguir participando en propuestas pedagógicas que propendan por el crecimiento cognitivo, artístico, comunicativo, corporal y socio afectivo de los niños y niñas.

Palabras clave: investigación cualitativa, habilidades comunicativas, estrategias pedagógicas, aprendizaje significativo, transferencia a la realidad. 


\section{Abstract}

Objective. To analyze how the preschool teachers from a public kinder-garden in Bogotá use the pedagogical strategies in their classes to stimulate the development of the communicative dimension, through a meaningful learning, which is transferable to their reality.

Methodology. This proposal is structured from a qualitative research, through the application of data collection instruments, such as: semi-structured interview, observation and content analysis, in a group of thirty-two infants, between four and five aged, and the teacher in charged, with the purpose of obtaining elements that were interpreted through the use of a data analysis strategy, to carry out with the analysis of data triangulation.

Results. Firstly, the impact of this project evidenced that designing and generating significant learning spaces where the children developed communicative skills which can be transferred to the reality, allowing them to perform with higher social level abilities. Secondly, with the implementation of pre-instructional, co-instructional and post-structural pedagogical strategies of a teaching-learning sequence, the development of skills and abilities in each dimension of the human being is stimulated, thus achieving an integral development which is useful and transferable to the students' reality.

Conclusion. The implementation of pedagogical strategies stimulates the development of communicative skills and competences, to generate active participation stages, where the collective construction of the knowledge allows the generation of a meaningful learning that can be transferred to the reality. The application of pedagogical strategies, also, the transmission of practical knowledge lead to the searching of a meaningful learning which exceeded the initial expectations and invited to continue participating in more pedagogical strategies that consolidate the cognitive, artistic, communicative, corporal and socio affective increasing in boys and girls.

Keywords: qualitative research, communication skills, pedagogical strategies, meaningful learning, transfer to reality. 


\section{INTRODUCCIÓN}

a sociedad de hoy en día avanza de manera Lertiginosa, a cada instante surgen desafíos que se deben asumir de manera inmediata. Los principales responsables en dar respuesta a las demandas del siglo XXI son los planteles educativos, al ser entes formadores mediante la implementación de modelos y estrategias que den solución a las problemáticas y realidades del presente. Es por ello, que se hace necesario un docente innovador, que generen un cambio continuo para estimular el desarrollo y la construcción del conocimiento, con base en el lenguaje y experiencias significativas que contribuyan a la transformación del contexto.

La escuela al ser una institución líder en la formación de personas, debe promover el desarrollo de habilidades comunicativas y de investigación, con el fin de crear escenarios de innovación y adaptación a los nuevos cambios, para instaurarse como principal agente de transformación social. Los integrantes de una comunidad educativa a través del lenguaje conocen el pensamiento y el accionar de las personas que se encuentran a su alrededor, permitiéndoles participar en una formación integral de los estudiantes. Para ello, es necesario a partir de lo que plantea McLaren (1995) desarrollar una actitud crítica frente a las ideas y prácticas culturales, en las cuales se encuentran inmersos.

Para Bar (1999), el docente al estar presente en un mundo de constantes cambios, debe generar acciones educativas que respondan de manera oportuna y positiva al proceso de enseñanza-aprendizaje del momento, teniendo presente que el aprendizaje debe ser el proceso por el cual las personas adquieren nuevas habilidades o conocimientos, con el propósito de mejorar su intelecto y calidad de vida. Por tanto, la enseñanza dentro de las instituciones debe reconocer que la labor educativa es un proceso de transformación mutua, donde el educador y el educando se desenvuelven como personas en la libertad, adquiriendo destrezas como: investigador, comunicador y creativo. Siendo, como lo afirmaba García (2001), el docente con su personalidad y habilidades, el que promueva el cambio, de la mano de todos los integrantes de la institución en la que labora. Permitiendo obtener a corto y largo plazo cambios que permitan el mejoramiento y la transformación de la educación.
El papel que cumple la escuela en la sociedad según Tedesco (2003), le exige la implementación de nuevos pilares que permitan dar respuestas oportunas a los cambios a nivel social, al asumir el reto en las aulas escolares de aprender a aprender y aprender a vivir juntos, enmarcados dentro del desarrollo cognitivo y socio afectivo de los estudiantes, consiguiendo con ello la formación de personas competentes al tener la capacidad de transformar su contexto inmediato a través de la interacción con los demás; con el propósito de proyectar acciones educativas encaminadas a producir e incrementar el conocimiento, para sentar bases duraderas, que trasciendan a nivel educativo al tener presentes las diversas perspectivas pedagógicas a largo plazo, según la Asociación Nacional de Universidades e Instituciones de Educación Superior (2004). Esto en palabras de Moschen (2005) sería la capacidad de proyectar acciones educativas encaminadas a liderar innovaciones pedagógicas para el mejoramiento institucional, teniendo presente el contexto histórico y los escenarios de futuro, con el objetivo de preparar a los estudiantes para ser competentes en diferentes campos de acción.

De ahí la necesidad de la actualización docente, idea apoyada por Torrecilla (2006), al establecer la formación pedagógica como medio para la creación de proyectos investigativos innovadores que den paso a la construcción de nuevas estrategias y métodos que transformen la cotidianidad a través de las prácticas pedagógicas críticas implementadas por los docente dentro del proceso de enseñanza-aprendizaje; al tener presentes diversos factores que influyen de manera notable en la evolución del mismo, tales como: el contexto social, el lenguaje, los avances tecnológicos, los conocimientos previos, los intereses y las necesidades de los estudiantes.

El Ministerio de Educación Nacional, el Ministerio de protección Social y el ICBF (2006), en el marco de la Política educativa para la primera infancia estableció que es deber del docente brindar experiencias significativas de aprendizajes a través de la implementación de diferentes ambientes socializadores que permiten el desarrollo integral de los estudiantes, mediante los pilares de la educación inicial: juego, arte, literatura y exploración del medio. Utilizando el lenguaje y la interacción, como procesos que permitan construir nuevas formas de pensar, sentir y expresar, con el fin que los infantes de- 
sarrollen habilidades comunicativas y expresen sus ideas, a partir de sus costumbres culturales, problemáticas sociales y peculiaridades individuales, idea planteada por el MEN (2006). Tomándose así, el aprendizaje como algo que se da al relacionarse con los demás, mediante el uso de diversas prácticas sociales y comunicativas, que dan como resultado diversos discursos que nacen en los diferentes escenarios donde está la persona, permitiéndole el intercambio de ideas y la construcción de sentidos.

De Zuburía (2006), hace reflexionar sobre cómo a lo largo de la historia la educación ha implementado diversos modelos pedagógicos que han permitido un gran avance tanto en la intervención con los integrantes de la comunidad educativa, como en la formulación de estrategias de organización y en el estudio de las condiciones de enseñanza y aprendizaje. Estos aportes han surgido a través de la historia pretendiendo dar respuesta a las necesidades de su momento, es así como hacia 1910 surge la teoría conductista, la cual ha desarrollado abundante conocimiento en el área de la educación y lenguaje. Teniendo diferentes autores que dieron aportes significativos; uno de ello es Skinner quien "entiende el lenguaje como el producto de un simple mecanismo de condicionamiento responsivo gradual reforzado. Se aprende a través de procedimientos de condicionamiento operante. La conducta verbal es un comportamiento reforzado, articulado a la mediación que se establece entre personas" (Palmero, 2006, p.40). Por lo que, la experiencia de aprendizaje para esta corriente implica la manipulación de aspectos observables del comportamiento, y estos se comprenden en los cambios de las condiciones ambientales.

A mediados del siglo XX, el aprendizaje se enfoca con una mirada constructivista, la cual propone condiciones del aprendizaje que concuerdan con la posibilidad de dominio y uso que cada persona puede tener de los conocimientos que adquiere, según lo explicado por De Zuburía (2006). Este autor, presenta la definición de constructivismo como algo variable de acuerdo al contexto, sin embargo, se comparte el principio de elaboración que realizan las personas de lo que observan o manipulan. Desde una postura centrada netamente en la Psicología cognitiva, se puede determinar que todo lo que se aprende y se sabe, es gracias a la experiencia y el lenguaje, ya que éste es el que permite conocer, comprender y compartir las ideas y conocimientos con los demás en un contexto real. Pérez y Salmerón (2006) aportan a esta idea, que el estudiante debe adquirir parámetros que le permitan desenvolverse en su entorno, a partir de la necesidad de desarrollar habilidades comunicativas, además que esto no solo les ayuda a adquirir conocimientos sino que contribuye a su proceso de socialización, permitiéndoles conocer el contexto dentro del cual viven para comunicarse con otros.

Las investigaciones empíricas señaladas con anterioridad, evidencian la importancia que a lo largo de la historia ha tenido el lenguaje en el desarrollo integral de todo ser humano, como principal proceso que permite la socialización y construcción de saberes, si se brinda un ambiente abierto a responder diversas ideas por medio de la expresión verbal, corporal, escrita, gestual y proxénica.

\section{LA NIÑEZ Y EL LENGUAJE EN EDUCACIÓN PREESCOLAR}

"Al niño para su desarrollo en los aspectos biológico, cognoscitivo, sicomotriz, socio-afectivo y espiritual, se le estimula a través de experiencias de socialización pedagógicas y recreativas" (Ley 115, artículo 12, p. 5). Las Instituciones escolares de preescolar tienen como principal misión, el brindar atención integral al niño y la niña, al ser garantes y defensoras de los derechos de esta población; acogiéndose a la Ley No. 12 del 28 de enero de 1991 donde se reconoce a los niños y a las niñas como sujetos sociales, ciudadanos con derechos en contextos democráticos; favoreciendo su desarrollo armónico integral, que considera aspectos físicos, psíquicos, afectivos, sociales, cognitivos y espirituales.

Cervera (2003), en su libro Adquisición y desarrollo del lenguaje en preescolar y ciclo Inicial, nombra la importancia de estimular el desarrollo del lenguaje en el preescolar, como un proceso natural; el cual requiere de la realización de actividades lúdicas que logren la asimilación y construcción de saberes, al participar en actividades creativas y de imitación, que le permitan al infante ir descubriendo el significado del mundo, para con ello poder accionar dentro de un proceso comunicativo, en donde el intercambio de ideas le permitan formarse como un ser activo dentro de un contexto sociocultural, en donde lee la realidad, la analiza, la comprende y expresa su 
sentir frente a esta, compartiendo sus emociones, pensamientos, sentimientos y necesidades, al construir de manera constante ideas con sus pares y docentes, ideas que le permiten darle un significado a su entorno.

El Fondo de la Naciones Unidas para la Infancia y la Universidad Especializada de las Américas (2004), en la guía de lenguaje y comunicación para la niñez de cuatro a seis años, nombra los aspectos mínimos que debe comprende el programa de preescolar en el área de lenguaje. En este documento presentan la importancia de estimular el desarrollo de las diferentes formas del lenguaje en los niños y niñas que asisten al preescolar, con el fin de capacitarlos para una comunicación asertiva que esté compuesta por las estructuras básicas del español como lengua materna, al aprovechar diversos recursos como: "emisión de sonidos espontáneos, la articulación de palabras y frases, narración de experiencias vividas, imitación de rimas, trabalenguas, retahílas, adivinanzas, la generación de familias de palabras desde un palabra de origen y ejercicios grafomotores, entre otros" (Fondo de la Naciones Unidas para la Infancia y la Universidad Especializada de las Américas, 2004, p.214).

Esta guía curricular para la niñez de cuatro a seis años, estructura el lenguaje en cuatro subáreas: lenguaje corporal, lenguaje, oral, lenguaje escrito y literatura; las cuales al ser trabajadas permite el alcance de los objetivos generales propuestos para esta área, los cuales son:

- Desarrollar el lenguaje como medio de comunicación, expresión y representación del pensamiento.

- Estimular las capacidades de expresión espontánea de lenguaje verbal y corporal.

- Desarrollar la senso - percepción del mensaje de la comunicación.

- Familiarizar a la niñez con la utilización del lenguaje oral y escrito.

- Promover el disfrute del mundo imaginario de la lectura”. Según el Fondo de la Naciones Unidas para la Infancia y la Universidad Especializada de las Américas, (2004, p.214).

En Bogotá (Colombia), las instituciones educativas siguen el modelo pedagógico llevado a cabo en el Instituto Alberto Merani, catalogado como la mejor institución educativa de la capital y la quinta a nivel nacional, por los excelentes resultados que obtienen los estudiantes en las pruebas saber (Vega, 2014). Julián de Zuburía, director de esta institución, resalta la importancia de educar en torno al lenguaje dentro del proceso de enseñanza-aprendizaje, ya que este permite el apropiamiento del conocimiento y la comprensión del entorno con una mirada crítica (Zuburía, 2013), en el área de lenguaje, presenta un enfoque integral, general y contextual por competencias desde las dimensiones del ser humano; enfoque que da paso a la creación y estimulación del desarrollo de las competencias comunicativas, en donde se ponen en juego la razón, la interlocución, la socialización, el saber hacer cultura y conocimiento a través del diálogo.

\section{ApRendiZAJe sIGNIFICATIVO}

Ormrod (2005), expone como día a día los diversos estudios arrojan cosas nuevas sobre el Aprendizaje Humano, un proceso maravilloso y enriquecedor pero desconocido por muchos. El aprendizaje, es el resultado de la recopilación y la adquisición de ciertos conocimientos, habilidades, destrezas, emociones y valores que le permiten al ser humano adaptarse, a través de la exploración al medio en el cual debe desenvolverse según sus necesidades, intereses y exigencias sociales.

El aprendizaje significativo es una propuesta pedagógica creada por David Paul Ausubel, quien junto a Novak y Hanesiana (2009), explica como el ser humano tiene la habilidad de enlazar los conocimientos previos obtenidos con la información nueva para poder construir saberes nuevos, haciendo de este modo en el campo educativo, que el estudiante sea el centro de su aprendizaje, teniendo en cuenta sus ideas previas y su contexto. Lo cual, según Ormrod (2005), le permitirá al estudiante encontrarle sentido a lo que aprende, apoderarse de nuevos conocimientos y a través del significado descubrir la razón de ser de lo que el maestro le presenta; permitiéndole actuar y dar solución a situaciones que se le presenten en el día a día.

Rodríguez (2008) en su libro: La teoría del aprendizaje significativo en la perspectiva de la psicología cognitiva, presenta el aprendizaje significativo desde una visión cognitiva. Al enseñar, como la teoría del aprendizaje propuesta por Ausbel da cuenta de los procesos cognitivos implicados en la interacción que se produce en- 
tre profesor, alumnos y materiales educativos, cuando se presenta y se adquiere una nueva información. Esta teoría aporta elementos esenciales que deben ser considerados en el proceso de enseñanza - aprendizaje, para garantizar que los nuevos conocimientos prevalezcan mucho mejor a la estructura cognitiva del ser humano.

\section{EstRATEGIAS PEDAGóGICAS}

Díaz (2009) en su libro Estrategias docentes para un aprendizaje significativo, plantea las estrategias de enseñanza como los procedimientos que el agente de enseñanza utiliza en forma reflexiva y flexible para promover el logro de aprendizajes significativos en los alumnos; son los medios o recursos para prestar la ayuda pedagógica. Propone para la realización de una estrategia de enseñanza tener en cuenta cinco aspectos esenciales para así considerar qué tipo de estrategia es la indicada para utilizarse en ciertos momentos de la enseñanza:

- Consideración de las características generales de los estudiantes.

- Tipo de dominio del conocimiento en general y del contenido curricular en particular, que se va a abordar.

- La intencionalidad o meta que se desea lograr y las actividades cognitivas y pedagógicas que debe realizar el alumno para conseguirla.

- Vigilancia constante del proceso de enseñanza

- Determinación del contexto intersubjetivo (por ejemplo, el conocimiento ya compartido) creado con los alumnos hasta ese momento, si es el caso.

Díaz (2009), señala que entre las estrategias de enseñanza más representativas, están: los objetivos, los resúmenes, los organizadores previos, las ilustraciones, los organizadores gráficos, las analogías, las preguntas intercaladas, las señalizaciones, los organizadores textuales, los mapas y redes conceptuales. El texto de Díaz (2009), explica como las anteriores estrategias se pueden incluir al inicio (preinstruccionales), durante (coinstruccionales) o al término (postinstruccionales) de una sesión, episodio o secuencia de enseñanza- aprendizaje:

- Las estrategias preinstruccionales preparan y alertan al estudiante en relación con qué y cómo va a aprender; activando el conocimiento y las experiencias previas pertinentes.

- Las estrategias coinstruccionales apoyan los contenidos curriculares durante el proceso mismo de enseñanza-aprendizaje.

- Las estrategias postinstruccionales se presentan al término del episodio de enseñanza y permiten al alumno formar una visión sintética, integradora e incluso crítica del material.

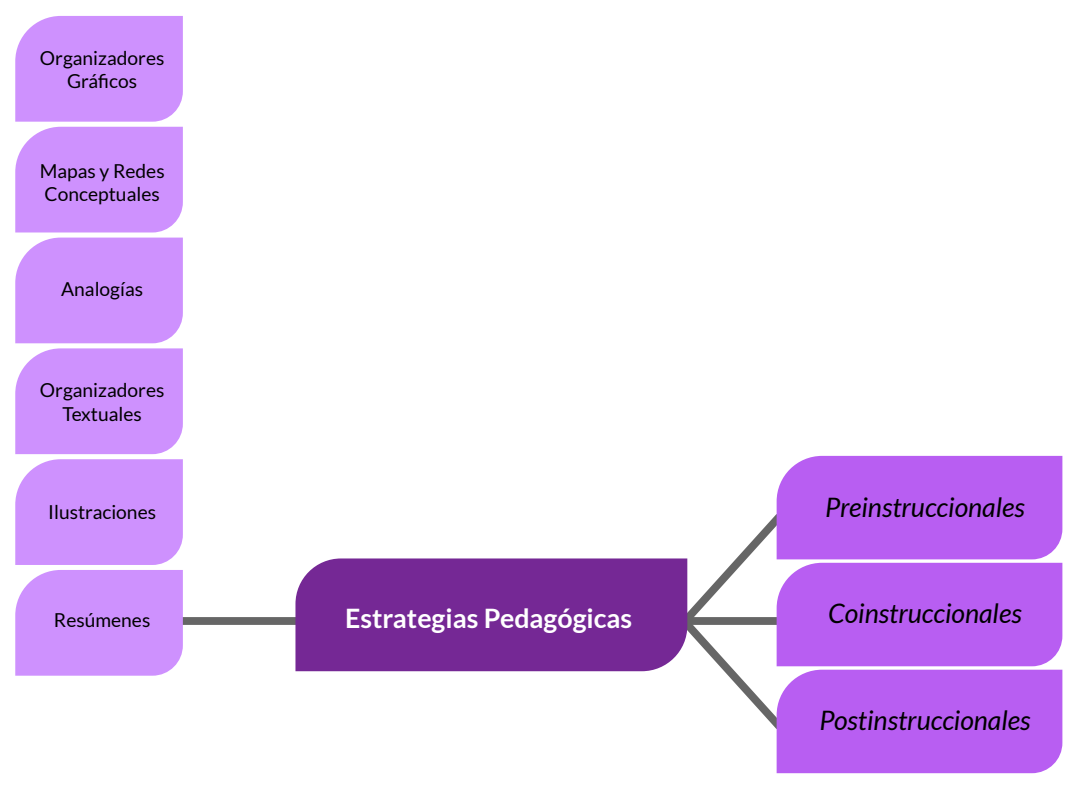

Tabla 1. Estrategias Pedagógicas.

Fuente: creación propia. 
Ormrod (2005), en este aspecto, hizo énfasis sobre la importancia que tiene, el que los profesores pueden ayudar a los estudiantes a dominar los conceptos en el aula si se centran en factores que facilitan el aprendizaje de conceptos por medio de esquemas, al ser ideas conectadas y relacionadas entre sí, por un acontecimiento en común. El aprendizaje del ser humano depende de muchas situación y herramientas utilizadas por el docente, las cuales le permiten al estudiante conocer en escenarios significativos, en donde por medio de experiencias vitales se le halle significado, utilidad y por ende aplicabilidad a lo estudiado, siendo factores motivantes y por ende determinantes dentro de un proceso de enseñanza-aprendizaje.

Trueba (1994), propone la idea de crear espacios dentro del aula de preescolar, en dónde los niños y las niñas tengan la oportunidad sonreír, quererse, descubrir, existir, ser y estar, en un sitio donde hallen respuestas a sus necesidades: físicas, socio afectivas, de independencia, de interacción, de pensamiento, de juego, de expresión, de experimentación, de descubrimiento. De este modo, el aula se vislumbra como el lugar de origen de infinidad de experiencias que dan paso a aprendizajes significativos, como una estrategia pedagógica y como una herramienta que respalda el proceso de enseñanza - aprendizaje, al propiciar el intercambio de ideas, información y recursos. A través del ambiente los docentes pueden dar origen a muchos medios de correlación que promuevan la construcción de nuevos saberes.

Díaz y Hernández (2002), presentan algunas estrategias de enseñanza que el docente puede utilizar con el propósito de facilitar el aprendizaje significativo de los estudiantes, estas son: objetivos o propósitos del aprendizaje, resúmenes, ilustraciones, organizadores previos, preguntas intercaladas, pistas topográficas y discursivas, analogías, mapas conceptuales y redes semánticas y el uso de estructuras textuales. Las cuales pueden incluirse antes, durante o después de un contenido curricular específico.

\section{LA TRANSFERENCIA EN EL APRENDIZAJE}

Wenzelburger (2013), escribe como al tratar de definir los propósitos de la enseñanza, surgen diversas posiciones y opiniones por parte de los docentes ante la temática, opiniones que llegan a un mismo punto de vista: la enseñanza brindada debe ser útil para el estudiante fuera de la institución educativa, el estudiante debe aprender para la vida, para responder a las diferentes demandas interpuestas por la sociedad. Para los docentes es fundamental la practicidad de ciertos conocimientos, los cuales los convierten no sólo en útiles para la vida de los estudiantes, sino también en llamativos en tanto su utilidad es clara. El docente al enseñar muestra su principal interés por transferir lo aprendido. Cuando Wenzelburger (2013) habla de transferencia, se refiere a lo aprendido y la posterior facilidad o inhibición que ocurre en otras situaciones de aprendizaje o desempeño. Todo aquello que se puede aprender es transferible, existen elementos cognoscitivos transferibles como: las habilidades psicomotoras, habilidades cognoscitivas, hechos, conceptos, principios, relaciones, teorías, estructuras, métodos para resolver problemas, etc.; al igual que actitudes y posiciones afectivas que pueden ser transferidas.

De ahí la importancia nombrada por Díaz y Hernández (2002), sobre la necesidad que tiene la escuela y la sociedad, de formar docentes que tengan el interés y la capacidad de incluir la realidad del contexto inmediato al ámbito educativo, para enfrentar los retos y demandas existentes en la sociedad de la cual hacen parte. La docencia es un proceso integral en el que el estudiante necesita recibir la estimulación necesaria para desarrollar habilidades comunicativas, socioafectivas, éticas y cognitivas, que le permitan crecer como persona al pensar, aprender y sentir. Frente a este aspecto, Sebastiani (2004) le da gran importancia dentro del proceso de enseñanza-aprendizaje a las acciones realizadas por parte del docente, en donde se creen los escenarios necesarios para lograr niveles de criticidad y reflexión que se convierten en sustento sobre el cual se genera toda una pedagogía que conjugada con los niveles académicos aprendidos, permita la formación integral de los estudiantes. Díaz y Hernández (2002) al igual comparten la idea de enlazar los saberes teóricos con la experiencia práctica para generar programas aplicables y reflexivos, que sean funcionales para el estudiante en los diferentes contextos.

\section{LA DIMENSIÓN COMUNICATIVA}

Los abundantes estudios sobre el lenguaje, dan muestra de su importancia para el desarrollo 
del ser humano. Al ser el proceso que posibilita el intercambio de ideas y la construcción de significados del contexto en el que se desarrollan las personas, por medio de la mutua comunicación a nivel individual, grupal y social. A nivel educativo "los profesores como intelectuales han de contemplarse en función de los intereses ideológicos y políticos que estructuran la naturaleza dentro del discurso, las relaciones sociales del aula y los valores mismos que ellos legitiman en su enseñanza” (Giroux, 1990, p.171).

González (2007), en su tesis doctoral realiza un análisis sobre la construcción conjunta del conocimiento a partir de debates postnarrativos, en donde nombra la importancia de crear historias con los niños y las niñas, para introducirlos en un mundo conceptual distante y más complejo dentro de un juego simbólico creativo, desarrollando notablemente la capacidad de uso del lenguaje. La estructura literaria de una narración puede dar vida a una conversación, posibilitando el debate, la comunicación de doble vía y modelos de forma en las que pueden discutir la literatura para intercambiar, asociar ideas y mejorarlas, dando una mejor interpretación.

\section{INTEGRACIÓN DE CONCEPTOS TEÓRICOS}

La escuela, por ser una institución social, no puede ser ajena a los requerimientos y realidades actuales de las personas que la conforman, es por ello que debe centrar su atención en las problemáticas que aquejan a niños y niñas del país e instaurar estrategias pedagógicas que le permitan a los infantes participar de manera activa en la realización de actividades, mediante el uso del lenguaje y de sus conocimientos previos, para interactuar, intercambiar ideas y construir nuevos saberes significativos, que contribuyan a la transformación social.

Las instituciones educativas coordinadas por los lineamientos curriculares establecidos por el MEN, buscan brindar una educación que aporte al desarrollo de un ser integral, al tomarlo en cuenta con una persona activa que requiere de escenarios que le permitan potencializar diversas destrezas y habilidades en la parte socio afectiva, cognitiva, comunicativa, corporal y artística. Dando utilidad y sentido a diferentes procesos educativos que le ayudarán al estudiante, a construir un pensamiento crítico y una necesidad inminente de crear estrategias que aporten a la transformación de las problemáticas que aquejan la sociedad, mediante la implementación de escenarios que permitan la socialización de ideas en un ambiente significativo y transformador, que puedan ser posibles, si las instituciones educativas recobran, el verdadero sentido de la educación, mediante el uso de una pedagogía que tenga como base el diálogo y el desarrollo de habilidades y destrezas comunicativas, necesarias para participar en el intercambio de opiniones, pensamientos y demás, que promuevan un accionar en busca de la transformación de la realidad a través del lenguaje, como principal medio socializador.

En este aparte, cabe mencionar los aportes de Ruíz (2000), en donde posiciona la palabra como la primera herramienta de relación del niño con el medio que le rodea. A través de ella interactúa y descubre, comunica y expresa todo lo que conoce y desea conocer. Es así como en las aulas se deben implementar propuestas pedagógicas que incentiven el uso del lenguaje en los niños y niñas, como pieza fundamental para crear y expresar las diferentes percepciones que tienen del mundo. Esto se logra con la elaboración de actividades de integración, que tienen en cuenta el bagaje de conocimientos y experiencias que trae el estudiante en su recorrido por el mundo del saber.

La revisión literaria propone el estimular el desarrollo de habilidades en los infantes de preescolar para que lleguen al saber hacer en un contexto, influido por las competencias comunicativas que comprenden: el acto de leer como un proceso significativo y semiótico, que va más allá de la pesquisa de significado, el acto de escribir como un proceso individual y social en cuanto se configura un mundo y se usan diversos saberes, competencias e intereses determinados por un contexto sociocultural; los actos de escuchar y hablar, en donde al hablar la persona piensa en función de la significación y la producción de sentido y la escucha, que implica ir construyendo el significado de manera inmediata, reconociendo la intención del hablante y el contexto social, cultural e ideológico.

\section{MÉtodo}

La misión de formar personas competentes e innovadoras, requiere por parte del docente la realización de procesos sistemáticos de explo- 
ración en la comunidad, en la institución y en el aula, significando conceptos, asumiendo nuevas estrategias, nuevas estructuras organizacionales, en busca de nuevos caminos que permitan construir aprendizajes a través de la experiencia. Aprendizaje que surge al implementar la investigación cualitativa, como un recurso primordial para lograr una aproximación a la realidad (Ruíz, 2012), que permite comprender el estudio de una realidad y realizar una construcción de sentidos y significados que se tejen en la interacción permanente entre los diferentes actores del proceso educativo.

El proyecto de investigación consiste en conocer las estrategias pedagógicas implementadas por el docente para el desarrollo de habilidades y destrezas propias de la dimensión comunicativa con niños y niñas de transición, que sean transferibles a la realidad mediante un aprendizaje significativo, idea que compagina con la investigación cualitativa, en la que prima el lenguaje, como encargado de configurar la realidad. Motivo por el cual, la investigación cualitativa es el método guía en el desarrollo del proceso, pues "El objetivo de la investigación cualitativa es la comprensión, centrando la indagación de los hechos...se pretende la comprensión de las complejas interrelaciones que se dan en la realidad" (Rodríguez, Gil y García, 1996, p.45). Este estudio tiene en cuenta las características principales de la investigación cualitativa nombradas por Strauss y Corbin (2002), al tomar: la realidad construida como un proceso de interacción con la misma; partir de una realidad específica para llegar a una teoría; la utilización de herramientas, estrategias y metodologías con el propósito de realizar una representación muy precisa de la realidad, objeto de investigación.

El enfoque cualitativo, permite el estudio con profundidad de situaciones concretas, mediante la comprensión e interpretación de las acciones realizadas en un contexto determinado, para servir como base, en el estudio del ambiente socio-cultural y en el diseño de estrategias de acción educativa. Según Pelekais (2000), el enfoque cualitativo tiene un claro fundamento humanista para abordar la comprensión y entendimiento de la realidad social, al resaltar la concepción progresista y negociada en la muestra de estudio, y señalar que la realidad no es estática, sino cambiante y dinámica; de la misma manera, las personas son concebidos como agentes activos en la construcción y definición de las realidades que las rodean, con lo cual su participación es decisiva en la comprensión de las situaciones salientes del contexto.

Para el desarrollo de la investigación cualitativa en el Jardín Infantil, se tuvo presente el tópico de interés: la vida cotidiana, la práctica educativa y experiencias significativas, cuyo fin es el mantener la atención por parte de la investigadora para hacer del mismo, un proceso de autorreflexión y crítica. "Los procesos, al igual que los diseños de investigación cualitativos, a menudo emergen de la reflexión del investigador tras sus primeras aproximaciones a la realidad objeto de estudio" (Rodríguez, Gil y García, 1996, p.61).

\section{Participantes de la inVestigación}

Para la investigación, participaron treinta y dos infantes que pertenecen al nivel de transición con edades entre los cuatro a cinco años de edad y la docente a cargo del curso, la cual cuenta con una formación profesional, al ser Licenciada en Educación Preescolar; la docente es la encargada de construir la malla curricular del área de español, explicarla a las demás docentes y velar por el cumplimiento de temáticas.

\section{TÉCNICAS E INSTRUMENTOS DE RECOLECCIÓN DE DATOS}

Las técnicas e instrumentos de recolección de datos permiten tener acceso a la información, mediante la cual se puede explorar, describir y explicar los hechos o fenómenos que definen el problema de la presente investigación. Los instrumentos utilizados, según Hernández, Fernández y Baptista (1998), deben ser confiables y válidos, para poder hacer con los datos hallados un análisis objetivo que aporte a la solución del problema.

\section{TÉCNICA DE LA OBSERVACIÓN}

La observación como técnica de recolección de datos demanda de un diseño de trabajo para captar las expresiones y aspectos más trascendentes y significativos del escenario de investigación, según Rojas (1980). En éste estudio se aplica la observación no participante, en la que el investigador no interviene en los quehaceres cotidianos que los infantes y docente desarrollan. Este tipo de observación se realiza en visitas programadas 
para reconocer y delimitar el área de estudio, con el fin de obtener información para poder ajustar hipótesis, responder las preguntas problema y adoptar estrategias en la aplicación de las demás técnicas que se utilizaran en la investigación, si es necesario.

\section{ANÁLISIS DE CONTENIDO}

Según Andréu (1998), es una técnica que permite analizar e interpretar textos, ya sean escritos, grabados, pintados, filmados, para conocer diferentes elementos y fenómenos de un contexto social. Porta y Silva (2003), lo enmarcan como una técnica objetiva, sistemática y cualitativa, mediante materiales representativos, señalados por la exhaustividad y la generalización. Al ser:

Objetiva: Utiliza procedimientos de análisis representados por otras investigaciones. Los resultados adquiridos pueden ser verificados por otros estudios distintos.

Sistemática: Requiere la contención del análisis a unas pautas objetivas determinadas.

Cualitativa: Muestra la presencia y ausencia de una cualidad del contenido y hace control de datos secundarios descritos a fenómenos a los que es posible referirse.

Representativa: Distingue materiales y categorías que pueden ser justificados.

Exhaustiva: No puede olvidarse nada del objeto de estudio escogido.

Generalización: Tiene unas hipótesis que deben ser probadas para plantear las conclusiones en la investigación.

\section{LA ENTREVISTA SEMIESTRUCTURADA}

La entrevista desde Valles (1997), es un constructo comunicativo cara a cara entre el investigador y el entrevistado para extraer información respecto a experiencias, vivencias o conocimientos, relatados con las propias palabras del interrogado, con el fin dar a conocer su experticia en el tema sobre el cual se está indagando. Según Vela (2001), el entrevistador mantiene la conversación dirigida sobre un tema particular, y le suministra al informador la autonomía suficiente para puntualizar el contenido de la discusión.

\section{El PROCEDIMIENTO DE LA INVESTIGACIÓN}

El proceso implementado para el desarrollo del proceso investigativo es el propuesto por Monje (2011), el cual está compuesto por cuatro fases, figura 1. Estas cuatro fases están acompañadas por etapas de la investigación cualitativa:

Fase de Solicitud: se presentó una carta de solicitud y consentimiento (Apéndice C) para realizar la aplicación de los instrumentos de recolección de información para el estudio dentro del aula de transición en el Jardín Infantil, junto con el propósito de la guía de observación y las fechas en que se realizara la observación de las clases. Se informará que la espectadora no intervendrá en el quehacer pedagógico de la docente, pues solo observara y diligenciara la guía de observación no participativa.

Fase Preparatoria: está compuesta por dos etapas: la reflexiva y de diseño. En la primera la investigadora, con base en sus conocimientos, experiencias, consultas e ideologías, construyó el marco teórico-conceptual, con el que se da inicio al proceso investigativo. En la etapa de diseño, se planificaron las actividades de las siguientes tres fases.

Fase Trabajo de campo: se accede al campo, para aplicar las diferentes técnicas de recolección de información, para después de obtener los suficientes datos del estudio investigativo, abandonar el campo. En esta fase se realizaron dos observaciones, las cuales tenían como finalidad, el analizar las estrategias pedagógicas implementadas por el docente de preescolar del Jardín infantil, para estimular el desarrollo de la dimensión comunicativa, a través de un aprendizaje significativo, que sea transferible a la realidad, durante el segundo semestre académico del 2015 .

Fase Analítica: se analiza con detenimiento y objetividad los datos recolectados en el trabajo de campo; para poder dar inicio a la obtención de resultados y confirmación de las conclusiones.

Fase Informativa: se termina el proceso de investigación con la presentación y difusión de los resultados obtenidos. 


\section{EstrategIA DE ANÁLISIS DE DATOS}

Se implementó la triangulación para confrontar la información obtenida en las diversas fuentes para darle claridad, veracidad y coherencia a la información recogida. Mediante el proceso fenomenológico se captaron significados y fenómenos en el contexto de estudio, a través de la utilización de herramientas convalidadas para la recolección de información como la entrevista semiestructurada, la observación no participativa y el análisis de documentos.

\section{Resultados}

Al analizar el instrumento de observación aplicado, se obtuvo que: En cuanto al uso de la estrategia pedagógica Proyecto de Aula, como recurso para desarrollar habilidades comunicativas en niños y niñas de preescolar, resultó bastante significativo para docente, padres y estudiantes que se pensara en la implementación de esta estrategia dentro del proceso de enseñanza- aprendizaje, ya que por sus características se muestra atractiva, novedosa y sencilla al momento de ser utilizada, mediante un interés patente en el deseo de ser partícipes de una experiencia educativa diferente, reflejado en el compromiso de todos y todas de cumplir con las tareas asignadas y participar activamente de los retos que se propondrán, con el fin de estimular el desarrollo de habilidades y destrezas comunicativas. Al observar y analizar lo logrado en cada una de las categorías, se evidencia el interés por participar en cada una de las actividades por parte de los estudiantes, al ser actividades lúdicas, llamativas, en donde los niños y niñas tienen la posibilidad de participar de manera activa en la construcción de saberes nuevos basados en los conocimientos previos aportados por todos y todas.

El incluir actividades artísticas, en este proceso de enseñanza aprendizaje, tanto padres como docente, reconocieron el valor que tiene el desarrollo de actividades artísticas dentro del proceso integral, formativo y de aprendizaje comunicativo de los niños y niñas, confirmando así por qué el arte ha sido escogido como pilar de la educación en la primera infancia, no centrándose en el resultado o producto sino fundamentándose en la oportunidad de expresión espontánea que hace un pequeño desde sus posibilidades, a través de la vivencia con otros y con el entorno, según la Secretaria Distrital de Integración so- cial (2013), situación que se evidenció en el desarrollo de las diferentes actividades planteadas dentro de la Clase de la observación 1.

Con lo observado se pudo deducir que el desarrollo de habilidades comunicativas se constituyó en un elemento esencial para la formación integral de los niños, puesto que con ellas pudieron elaborar una imagen más próxima de la realidad, a través de las diversas vivencias y experiencias que derivan de la relación con sus padres, docente y pares.

\section{ANÁLISIS DESCRIPTIVO E INTERPRETACIÓN DE LA MATRIZ DE ANÁLISIS DE CONTENIDO}

En el proyecto pedagógico, el Jardín Infantil busca propiciar un proceso comunicativo en los niños y las niñas, enriquecido mediante la socialización de forma significativa, ya que el Jardín busca vincular lo aprendido a la vida cotidiana de una manera libre y espontánea. El Jardín Infantil, cree que el niño y la niña desde su infancia, va complejizando y ligando su interés por relacionarse y aprender, gracias a las estructuras $\mathrm{y}$ formas de conocimiento que ya ha logrado o que están en pleno proceso de construcción, al participar de forma activa en cada una de las actividades propuestas, que tienen como principal finalidad: estimular el desarrollo del lenguaje verbal y no verbal con procesos corporales de escucha y habla.

El Jardín Infantil en su Proyecto Pedagógico, en el aparte de Estrategias Pedagógicas, busca ofrecerles a los niños y las niñas la posibilidad de conocer diferentes géneros literarios, a través cuentos, láminas, videos, títeres, audio cuentos, para que puedan descubrir y vivenciar el mundo imaginario de la literatura y diferenciar los recursos significativos de la lengua expresiva, logrando así enriquecer su vocabulario, y valorar a su vez el libro como portador de mensajes. De esta manera los infantes podrán: conocer, interpretar, investigar y vivenciar diferentes formas de expresión comunicativa, estimulando y enriqueciendo la creatividad, la imaginación y desarrollando el sentido artístico, al tener el arte como principal generador de habilidades y destrezas, en busca de un desarrollo integral en los infantes. El jardín Infantil, tiene muy claro y presente que la implementación de estrategias pedagógicas, aporta de manera notoria, al desarrollo de habilidades y destrezas comunicativas en los niños y niñas de transición. 


\section{ANÁLISIS DESCRIPTIVO E INTERPRETACIÓN DE LA ENTREVISTA SEMIESTRUCTURADA}

Con las respuestas dadas por la docente a las preguntas de la entrevista planteada, se pudo evidenciar como cada una de las actividades ofrecen y promueven las mismas oportunidades de desarrollo armónico e integral a todos los niños y niñas, al favorecer el desarrollo de su identidad, establecer condiciones y propuestas pedagógicas desde un enfoque de atención diferencial al brindar espacios de participación democrática y ciudadana que permitan la presencia de pilares como: el arte, el juego, la literatura y la exploración del medio, para propiciar experiencias pedagógicas que fortalezcan el desarrollo de cada una de las dimensiones a nivel socio afectivo, corporal, artístico, comunicativo y cognitivo, teniendo en cuenta las necesidades, diferencias, intereses, características y potencialidades de la población infantil, para promover con base a ello, el bienestar integral del niño y la niña, ampliando y diversificando las experiencias de aprendizaje y desarrollo, en un ambiente diseñado especialmente para el aprendizaje continuo y significativo.

La docente y el Jardín como tal, reconocen que los niños y niñas tienen todas las potencialidades para desarrollar y adquirir aprendizajes, mediante el juego, la fantasía y la percepción del mundo, logran entender lo que les sucede, lo que les rodea. Los niños y niñas, son concebidos como poseedores de unos saberes que han adquirido en sus relaciones consigo mismo, con las personas que le rodean, con los objetos, en sus vivencias y situaciones. En estas situaciones, lo que ven, escuchan y viven, les permite desarrollar nuevos conocimientos, modificar los que tenían y crear expectativas frente a otros. Lo anterior quiere decir que los niños siempre, desde que nacen, están en disposición de aprendizaje, y lo logran por sí mismos, pero en especial con la mediación de sus pares y adultos que le rodean.

La docente considera que los niños y las niñas de transición, tienen las máximas capacidades para aprender, construir, interpretar y proponer con una visión crítica acorde a su edad, sus relaciones, contexto y su posibilidad de poner en práctica el conocimiento en una situación determinada, en relación con los demás, y con permanente construcción.

\section{Discusión}

La aplicación de las herramientas de recolección de información, dejo conocer como el Jardín Infantil al ofrecer y promover las mismas oportunidades de desarrollo armónico e integral a todos los niños y niñas, favorece el desarrollo de su identidad, estableciendo condiciones y propuestas pedagógicas desde un enfoque de atención diferencial al brindar espacios de participación con la presencia de pilares como: el arte, el juego, la literatura y la exploración del medio, que propiciaron experiencias pedagógicas que fortalecieron el desarrollo de cada una de las dimensiones a nivel socio afectivo, corporal, artístico, comunicativo y cognitivo, teniendo en cuenta las necesidades, diferencias, intereses, características y potencialidades de la población infantil, promoviendo con base a ello el bienestar integral del niño y la niña, ampliando y diversificando las experiencias de aprendizaje y desarrollo, en un ambiente diseñado especialmente para el aprendizaje continuo.

La aplicación de estrategias pedagógicas además de la transmisión de un conocimiento práctico, permitió el logro de un aprendizaje significativo que sobrepasó las expectativas iniciales, e invitó a seguir participando en propuestas pedagógicas que propendan por el crecimiento cognitivo, artístico, comunicativo, corporal y socio afectivo de los niños y niñas.

Uno de los derechos fundamentales de los niños y niñas es la libertad de expresión y la participación activa en su desarrollo y el de su sociedad. Por tal motivo la docente del Jardín Infantil Arco Iris de Amor realiza asambleas diarias, en las que escucha la palabra de los niños y niñas para conocer sus ideas, iniciativas y aprender de ellos otras formas de ver el mundo para solucionar situaciones que se presentan en nuestra vida cotidiana.

La maestra hizo uso de la estrategia pedagógica: "Proyecto de aula", una estrategia que promovió la participación activa de cada estudiante, para la generación de nuevos saberes con base en saberes previos propios y de pares. Fue una estrategia que cultivo en los niños y las niñas el gusto por la lectura, por investigar, escribir y compartir lo aprendido al averiguar, ya que el proyecto de aula surge de las necesidades e intereses del niño y la niña por conocer y explorar experiencias nuevas y significativas.

La implementación de estrategias pedagógicas, motivo al estudiante a participar y construir su propio saber con base en sus conocimientos previos para con ello desarrollar diferentes habilidades y destrezas que le permitieron potencializar su proceso de enseñanza - aprendizaje 
Referencias bibliográficas

Andréu, J. (1998). Las técnicas de Análisis de Contenido: Una revisión actualizada. Granada, España: Ed. Universidad de Granada. Recuperado de: http://mastor.cl/blog/wp-content/uploads/2018/o2/Andreu.-analisis-de-contenido.-34-pagspdf.pdf

ANUIES, (2004). Documento estratégico para la innovación en la educación superior. Asociación Nacional de Universidades e Instituciones de Educación Superior. Recuperado en: http://comitecurricularsistemas.wikispaces.com/ file/view/INNOVACION_EN_LA_EDUCACION_SUPERIOR_ANUIES_MAYO+2004.pdf

Ausubel, D., Novak, J. y Hanesian, H. (2009). Psicología Educativa: Un punto de vista cognoscitivo. $2^{\circ}$ Edición. Distrito federal, México: Trillas. Recuperado de: https://edusique.wordpress.com/2011/11/11/psicologia-educativa-un-punto-devista-cognoscitivo-de-ausubel/

Bar, G. (1999, septiembre). Perfil y competencias del docente en el contexto institucional educativo. Trabajo presentado en el I Seminario Taller sobre Perfil del Docente y Estartegias de Formación. Lima, Perú: Organización de Estados Iberoamericanos. Para la Educación , la ciencia y la cultura. Recuperado de: https://es.scribd.com/ document/322600819/Perfil-y-Competencias-Del-Docente-en-El-Contexto-Institucional-Educativo

Cervera, J. (2003). Adquisición y desarrollo del lenguaje en Preescolar y Ciclo Inicial. Alicante, España: Biblioteca Virtual Miguel de Cervantes. Recuperado de: http://biblioteca.org.ar/libros/132266.pdf

Congreso Nacional de Colombia. (1991). Ley 12 de 1991. Convención Internacional sobre los Derechos del Niño. Bogotá, Colombia. Recuperado de: https://www.unidadvictimas.gov.co/sites/default/files/documentosbiblioteca/ley-12-de-1991. pdf

De Zuburía, J. (2006). Los modelos pedagógicos: hacía una pedagogía dialogante. Bogotá, Colombia: Cooperativa Editorial Magisterio. Recuperado de: http://bibliotecadigital.magisterio.co/libro/los-modelos-pedag-gicos-hacia-una-pedagogdialogante

Díaz, F. (2009) Estrategias docentes para un aprendizaje significativo. Distrito Federal, México: Editorial Trillas. Disponible en: http://www.alames.org/documentos/estrat.pdf

Díaz, F. y Hernández, G. (2002). Estrategias Docentes para un aprendizaje significativo. Una Interpretación Constructivista. Distrito Federal, México: Mc Gran Hill Interamericana. Recuperado de: https://jeffreydiaz.files. wordpress.com/2008/o8/estrategias-docentes-para-un-aprendizaje-significativo.pdf

Fondo de la Naciones Unidas para la Infancia y Universidad Especializada de las Américas. (2004). Guía Curricular de Estimulación temprana. o a 6 años. Ciudad de Panamá, Panamá: UNICEF y UDELAS. Recuperado de: http://files. unicef.org/panama/spanish/Guia-curricular-esti-temprana.pdf

García, L. (2001). ¿Dónde están las bases para las buenas prácticas en educación a distancia? Trabajo presentado en la Conferencia impartida durante el X Encuentro Internacional a Distancia. Guadalajara, México. Recuperado de: http:// scielo.sld.cu/scielo.php?script=sci_arttext\&pid=S0257-43142015000300006

Giroux, H. (1990). Los profesores como intelectuales. Barcelona, España: Paidós. Recuperado de: http://funama.org/data/ PEDAGOGIA\%20CRITICA/giroux/Los\%2oProfesores\%20como\%2oIntelectuales.pdf

González, J. (2007). Las narraciones y la tradición oral en el aula de preescolar. SUMMA Psicológica UST, 4(2), 129-136. ISSN: 0718-0446. Recuperado de: https://www.researchgate.net/publication/28223677_Las_narraciones_y_la_ tradicion_oral_en_el_aula_de_Preescolar

Hernández, R., Fernández, C. y Baptista, P. (1998). Metodología de la Investigación. Distrito federal, México: Editorial McGraw-Hill. Recuperado de: http://www.unipamplona.edu.co/unipamplona/portalIG/home_158/recursos/ebooks/16062015/metodologia.pdf

McLaren, P. (1995). Pedagogía crítica y cultura depredadora. Barcelona, España: Paidós. Recuperado de: http://funama. org/data/PEDAGOGIA\%20CRITICA/mclaren/Pedagogia\%2ocritica\%20y\%20cultura\%2odepredadora.pdf

Ministerio de Educación Nacional, Ministerio de Protección social e Instituto Colombiano de Bienestar familiar (2006). Política Pública Nacional de Primera Infancia. “Colombia por la Primera Infancia”. Bogotá, Colombia: Compes. Recuperado de: https://www.mineducacion.gov.co/1759/articles-177832_archivo_pdf_Conpes_109.pdf

Ministerio de Educación Nacional. (1994). Ley 115 de Febrero 8 de 1994. Bogotá, Colombia. Recuperado de: https://www. mineducacion.gov.co/1621/articles-85906_archivo_pdf.pdf 
Ministerio de Educación Nacional (2006). Plan Nacional Decenal de Educación 2006-2016. Lineamientos del PNDE para la educación preescolar, básica y media. Bogotá, Colombia. Disponible en: http://www.plandecenal.edu.co/html/1726/ articles-166057_prebasica.pdf

Monje, C. (2011). Metodología de la investigación cualitativa y cualitativa. Guía didáctica. Neiva, Colombia: Unisurcolombiana. Recuperado de: https://www.uv.mx/rmipe/files/2017/o2/Guia-didactica-metodologia-de-lainvestigacion.pdf

Moschen, J. (2005). Innovación Educativa: Decisión y búsqueda permanente. Buenos Aires, Argentina: Editorial Bonum. Recuperado de: revistas.ufro.cl/index.php/educacion/article/view/908/780

Ormrod, J. E. (2005). Aprendizaje humano (6 $6^{\mathrm{a}}$ Ed.). Madrid, España: Pearson, Prentice Hall. Recuperado de: http://www. visam.edu.mx/archivos/_LIBRO\%20Aprendizaje\%20humano_Ormrod\%204rta-ed_2004_PEARSON.pdf

Palmero, M. L. (2006, Enero/Junio). La teoría del aprendizaje significativo y el lenguaje La teoría del aprendizaje significativo y el lenguaje. Periódico do Mestrado em Educação da UCDB. Pp. 33-52. Disponible en: http://www.serieestudos.ucdb.br/index.php/serie-estudos/article/view/290/143

Pelekais, C. (2000). Métodos cuantitativos y cualitativos: diferencias y tendencias. Revista Telos, 2(2). Disponible en: http:// publicaciones.urbe.edu/index.php/telos/article/viewFile/1200/2491

Pérez, P. y Salmerón, T. (2006). Desarrollo de la comunicación y del lenguaje: indicadores de precaución. Revista de Pediatria y Atención Primaria, 8(32), 111-125. Recuperado de: http://archivos.pap.es/files/1116-612-pdf/637.pdf

Porta, L. y Silva, M. (2003). La investigación cualitativa: El Análisis de Contenido en la investigación educativa. Patagonia, Argentina: Universidad Nacional de la Patagonia Austral. Recuperado de: http://abacoenred.com/wp-content/ uploads/2016/o1/An\%C3\%A1lisis-de-contenido-en-investigaci\%C3\%B3n-educativa-UNMP-UNPA-2003.pdf.pdf

Rodriguez, G., Gil, J. y García, E. (1996). Metodoligia de la Investigación Cualitativa.Madrid, España: Ediciones Aljibe. Recuperado de: https://www.researchgate.net/publication/44376485_Metodologia_de_la_investigacion_cualitativa_ Gregorio_Rodriguez_Gomez_Javier_Gil_Flores_Eduardo_Garcia_Jimenez

Rodríguez, L. (2008). La teoría del aprendizaje significativo en la perspectiva de la psicología cognitiva. Barcelona, España: Editorial Octaedro. Recuperado de: http://cmc.ihmc.us/papers/cmc2004-290.pdf

Rojas, R. (1980). El proceso de investigación. Distrito Federal, Mexico: Trillas. Recuperado de: https://es.scribd.com/ doc/97099048/Raul-Rojas-Soriano-pdf

Ruiz, C. (2000). El juego lingüístico en la educación infantil. Revista Aula Abierta, (75). ISSN 0210-2773. Recuperado de: https://dialnet.unirioja.es/servlet/articulo?codigo $=45476$

Ruiz, J.I. (2012). Metodología de la investigación Cualitativa. Bilbao, España: Universidad de Deusto. Recuperado de: http://www.webquestcreator2.com/majwq/public/files/files_user/41661/o5.\%20Metodolog\%C3\%ADa\%20de\%20 la\%2oInvestigaci\%C3\%B3n\%20Cualitativa.pdf

Sebastiani, Y. (2004). Promoviendo el pensamiento crítico y creativo en la escuela. UMBRAL. Revista de Educación, Cultura y Sociedad, 4(7), 115-120. Disponible en: http://sisbib.unmsm.edu.pe/bibvirtualdata/publicaciones/umbral/vo4_no7/ A13.pdf

Strauss, A. y Corbin, J. (2002). Bases de la investigacióncualitativa. Técnicas y procedimientos paradesarrollar la teoría fundamentada.Medellín, Antioquía: Primera edición (en español): Editorial Universidad de Antioquia. Recuperado de: https://diversidadlocal.files.wordpress.com/2012/o9/bases-investigacion-cualitativa.pdf

Tedesco, J. C. (2003). Los pilares de la educación del futuro. Trabajo presentado en: Debates de educación. Barcelona, España. Disponible en: http://www.uoc.edu/dt/20367/index.html

Torrecilla, J. M. (2006). Modelos Innnovadores en la Formación Inicial Docente. Una apuesta por el cambio. Santiago de Chile, Chile: Unidad de Cooperación Internacional del Ministerio de Educación y Ciencia de España a las actividades del Proyecto Regional de Educación para América Latina y el Caribe (PRELAC) y la UNESCO. Recuperado de: file://C:/ Users/angie/Downloads/modelos_innovadores_formacion_inicial_docente.pdf

Trueba, B. (1994). El ambiente también educa. Revista de la Asociación Rosa Sensat, (24), 9-12. Recuperado de: https:// dialnet.unirioja.es/ejemplar/107604

Valles, M. (1997). Técnicas cualitativas de investigación social. Madrid, España: Editorial Síntesis. Recuperado de: https:// metodologiaecs.files.wordpress.com/2014/11/vallesmiguel-tc3a9cnicas-cualitativas-de-investigacic3b3n-social-1999.pdf 
Vega, A. (2014). Los seis mejores. Revista Dinero, (459), 18-20. Recuperado de: https://www.dinero.com/edicion-impresa/ caratula/articulo/los-mejores-colegios-colombia-2014/203553

Vela, Fortino. (2001). Un acto metodológico básico de la investigación social: la entrevista cualitativa. Distrito Federal, México: Porrúa. Recuperado de: https://periferiaactiva.files.wordpress.com/2016/o8/vela-peon.pdf

Wenzelburger, E. (2013). La transferencia en el aprendizaje. Distrito Federal, México: Anuies publicaciones. Recuperado de: http://publicaciones.anuies.mx/pdfs/revista/Revista61_S1A4ES.pdf

Zuburía, J.D. (2013). Instituto Alberto Merani. Disponible en: http://www.institutomerani.edu.co/principal/nosotros/ areas/lenguaje 
Revista incluida en los siguientes agregadores de contenidos
Revista incluida en los siguientes directorios

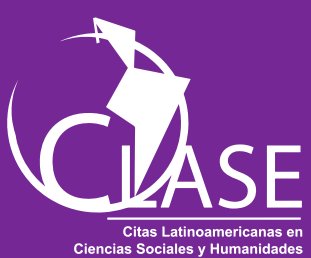

llboblat

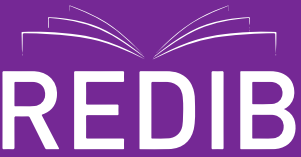

Red Iberoamericana

de Innovación y Conocimiento Cientifico

\section{Google Académico}
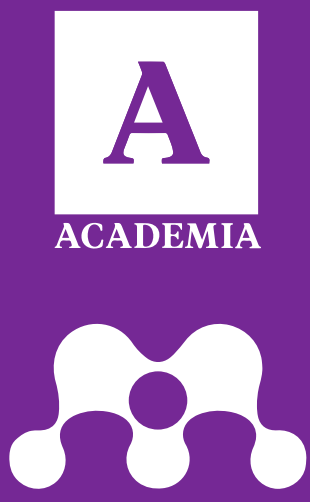

MENDELEY

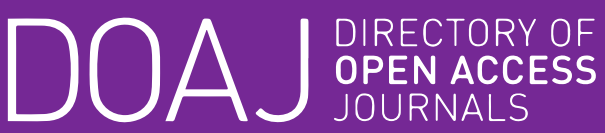

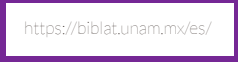
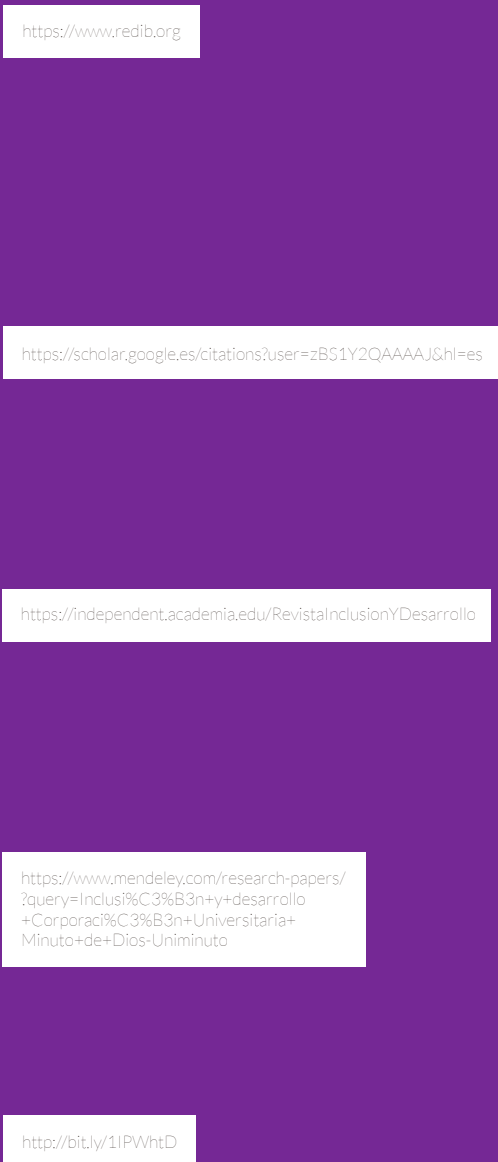

Revista incluida en la siguiente red social

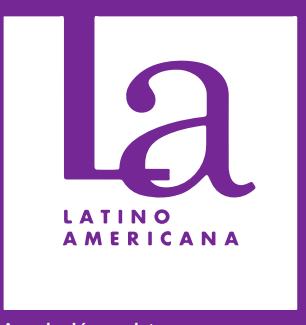



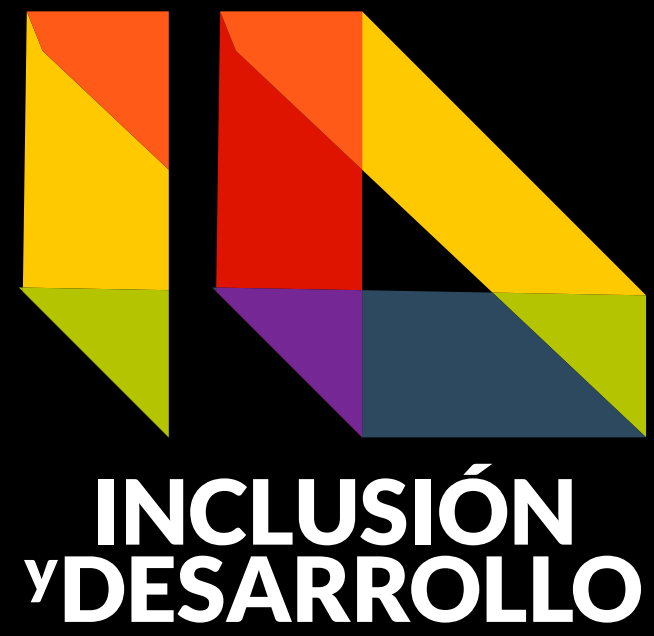

No. 1 Vol. 6 Año 2019 ISSN En línea: 2590-7700

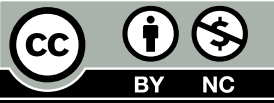

